\title{
Frontières
}

\section{Délires et dangers, mais surtout fascination et ravissement}

\section{Diane Laflamme}

Volume 15, numéro 1, automne 2002

Délires urbains, dangers de mort

URI : https://id.erudit.org/iderudit/1073899ar

DOI : https://doi.org/10.7202/1073899ar

Aller au sommaire du numéro

Éditeur(s)

Université du Québec à Montréal

ISSN

1180-3479 (imprimé)

1916-0976 (numérique)

Découvrir la revue

Citer ce document

Laflamme, D. (2002). Délires et dangers, mais surtout fascination et ravissement. Frontières, 15(1), 3-6. https://doi.org/10.7202/1073899ar d'utilisation que vous pouvez consulter en ligne.

https://apropos.erudit.org/fr/usagers/politique-dutilisation/ 


\section{DÉLIRES ET DANGERS, mais surtout fascination et ravissement}

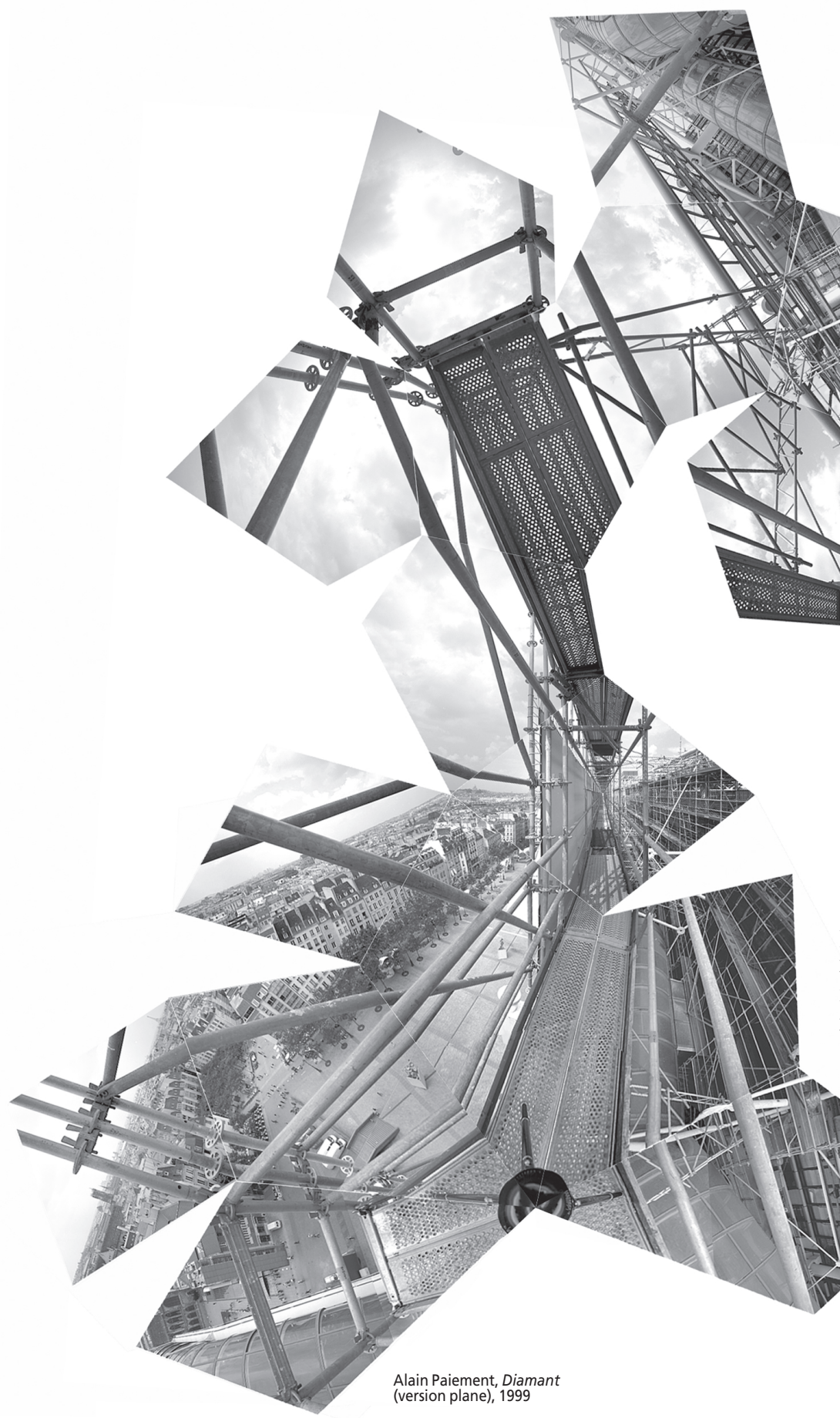

TU SAIS MON VIEUX FRANÇOIS, LA VILLE C'EST PAS POUR MOÉ RETOURNONS DANS NOTRE ÎLE, EMMĖNE MOÉ AVEC TOÉ ICITTE Y A TROP D'MACHINES, ÇA SENT LE RENFERMÉ ET PIS BONTÉ DIVINE, ON VOIT PAS NOS JOURNÉES. [...] J'VEUX PAS MOURIR EN VILLE TOÉ AUSSI BEN QUE MOÉ RETOURNONS DANS NOTRE ÎLE EMMĖNE MOÉ AVEC TOÉ.

MON VIEUX FRANÇOIS, PAROLES ET MUSIQUE DE LAWRENCE LEPAGE, ENFIN, 1976.
Diane Laflamme, Ph.D., rédactrice en chef.

Nos villes sentent-elles encore le renfermé comme le déclare en exergue la femme du vieux François? S'il est toujours vrai qu'on n'y voit pas nos journées, nous semblons nous en être fort bien accommodés; sommes-nous maintenant immunisés contre les courses et les essoufflements qui, pour plusieurs, font partie intégrante du mode de vie urbain? Bonté divine, il faut bien gagner sa vie avant d'en mourir!

Nous choisirons, pour la plupart, de vivre en ville pendant des périodes plus ou moins longues de notre existence à cause des études ou du travail, pour se rapprocher de la famille ou pour la fuir. Nous

laisserons souvent les circonstances orienter nos trajectoires... mais il nous arrivera aussi, peut-être pour échapper à l'odeur 
de renfermé, de remuer ciel et terre pour arracher des plages de notre agenda aux tentacules du centre-ville et retrouver "notre île » créée selon nos moyens à même les fleurs du balcon, le vert bruissant des soirées dans des banlieues boisées ou encore les fins de semaine dans une résidence secondaire enfouie loin du monoxyde de carbone. Même si nous nous installons en permanence dans une campagne qui nous est chère depuis l'enfance ou encore sur un îlot de sol découvert au fil de nos explorations, nos chemins n'en finiront pas de nous ramener en ville, pour le plaisir ou pour l'urgence.

Que nous aimions ou non vivre en ville, pourrons-nous vraiment refuser de «mourir en ville » comme l'épouse du vieux François? Soucieux de nous garder branchés à la vie le plus longtemps possible, ceux qui nous aiment réussirontils à nous convaincre de rester en ville ou d'y revenir, de choisir pour mourir des lieux où règnent en tandem technologie de pointe et épuisement professionnel poignant? Il se peut fort bien qu'on nous ramène en ville par compassion. Nous savons que les corridors des hôpitaux sont déjà pleins; quels visages se pencheront sur nous pour nous refuser droit de cité ? Penchés sur nous, il y aura : eux, avec leurs yeux haut perchés; en bas, il y aura: nous, objets balayés par leur regard méthodique. Ces yeux haut perchés seront-ils inspirés par la sollicitude ou par un souci de répartition efficace des ressources si utilement rassemblées en ville?

\section{REGARD HAUT PERCHÉ ET FAÇADES}

Nos lecteurs pourront s'exercer à pratiquer à leur tour une autre sorte de regard haut perché grâce à certaines des œuvres d'Alain Paiement présentées dans ce numéro. Qui aura reconnu dans Diamant, placé en première page de l'éditorial, le Centre Georges-Pompidou de Paris, photographié lors des récents travaux de rénovation? Si vous vous amusez comme moi à reconstruire le polyèdre après avoir découpé une copie de l'image, vous verrez apparaître un morceau de ville qui revêtira entre vos doigts une forme cristalline. L'œuvre a d'ailleurs été exposée sous cette forme également et figure au catalogue (planche 23).

Pour vivre l'expérience du regard haut perché, vous voudrez surtout vous attarder à des œuvres comme $F 3$ (Living Chaos), Local Rock et surtout l'installation Parages. L'effet est immédiat: nos pieds quittent instantanément sols, planchers et tapis. Haut perchés, nos yeux observent un lieu à la fois familier et déroutant. En regardant ces montages photographiques, la vitesse avec laquelle notre oil se repaît de la profusion des détails qui s'inscrivent en clair dans notre champ de vision est sans commune mesure avec la lenteur qui s'empare du même coup de tout ce qui en nous, muscles ou neurones, sert habituellement à nos déplacements dans l'espace.

Parages et Mute (tour silencieuse) faisaient partie de l'exposition d'Alain Paiement Le monde en chantier, organisée à la galerie de l'UQÀM en octobre et novembre 2002 par la commissaire Anne-Marie Ninacs. En circulant dans la galerie autour de l'installation Parages, le visiteur avait accès aux diverses couches de l'immeuble où habite l'artiste, à Montréal. Dans le catalogue de l'exposition, Anne-Marie Ninacs (p. 31) décrit le procédé : «l'appartement ici encore n'est pas sans lien avec l'univers puisque Paiement le remet dans son contexte, reconstituant la pâtisserie du rez-dechaussée de l'immeuble, une partie de la cave et du toit, l'espace de la rue et la tranquille cour arrière par rapport auxquels il le situe, créant ainsi un véritable microcosme qui se déploie en de gigantesques planchers installés dans l'espace les uns devant les autres, comme si l'édifice dans la galerie avait simplement été couché ». Plusieurs éléments de Parages sont reproduits dans nos pages : puits (p. 72-73), partir d'où j'habite (p. 55), pane mundial (p. 74-75) et cavel iris (p. 77). Décrivant pane mundial, Ninacs (p. 32) écrit: «L'appartement, autour duquel bourdonnent une foule de personnages en pleine activité - à la boulangerie certains préparent du pain, des gâteaux et des pizzas, un autre embarque la fournée quotidienne pour la livraison, des passants déambulent doucement sur le trottoir alors qu'un groupe s'est carrément emparé de la rue pour célébrer la victoire du Brésil au Mundial de foot -, apparaît aussi comme une petite enclave au sein de la ville en mouvement et exacerbe du coup la réalité troublante de la simultanéité de nos parcours ». Dans Parages, il y a un être, un seul, qui nous regarde droit dans les yeux : c'est un chat tigré, debout sur ses deux pattes arrière, les oreilles dressées, en suspension entre un haut et un bas qui se jouent directement à la verticale entre ses yeux et les nôtres au lieu de se figer, comme le voudrait la convention, entre le haut et le bas de la page imprimée.

"Le sage respire par les talons », disait Confucius ; quant à l'observateur haut perché, il a intérêt à découvrir où l'artiste a posé son talon. Dans Ocean Park par exemple, notre regard a besoin de ce pied pour ramener à l'échelle d'un coin de plage des courbes et des volumes qui pourraient bien nous donner l'illusion de survoler une ville toute entière.

Une œuvre plus ancienne d'Alain Paiement, Cassure (1997) (aux pages 26 et 27, avec un détail en page 22), nous ramène à une posture qui nous est plus familière : marcher en villes, côtoyer des façades. Pourtant, les piétons qui circulent sur ce trottoir bien ordinaire et les autos sagement stationnées aux espaces prévus sont entraînés à notre insu dans un curieux effet de transparence. Ces personnes, ces véhicules sont-ils vraiment là ? Ils sont doués d'une virtualité qui leur est salutaire compte tenu des débris qui s'écroulent sur eux. Tout cela à cause d'une béance dans la façade. Alain Paiement disait lors d'une entrevue en 1998: « la ville m'est apparue comme un ensemble de "devantures", qu'elles soient résidentielles, commerciales ou corporatives. Cet effet est accentué par ce qu'on appelle le façadisme, ce procédé de rénovation urbaine qui conserve les façades en reconstruisant totalement l'espace qu'elles cachent. Cette question de devanture amène une problématique de frontalité et de masque... J'ai voulu investiguer la ville en regardant ses surfaces architecturales, en portant mon attention sur certaines façades d'édifices "symptomatiques" de la ville en transformation, de cette disjonction entre la devanture et ce qu'elle dissimule».

En page couverture, avec des couleurs et des formes animées d'étranges mouvements qui amorcent des spirales, Alain Paiement travaille sur des façades trouvées à New York. Il s'agit d'un détail de l'œuvre Sometimes Square (version plane), présentée dans son entier aux pages 10 et 11 . Deux autres détails sont repris aux pages 57 et 61 . AnneMarie Ninacs commente aussi cette œuvre dans le catalogue publié en 2002, précisant entre autres que l'artiste y a rassemblé des vues de la $47^{\mathrm{e}}$ Rue datant de 1989 , d'autres prises entre les $44^{\mathrm{e}}$ et $45^{\mathrm{e}}$ Rues en 1991 et d'autres encore prises près de la $42^{\mathrm{e}}$ Rue en 1993. L'artiste a transformé les textes qui apparaissent sur les façades qui s'animent dans la nuit. Ninacs (p. 20) explique : «Bon nombre des enseignes lumineuses qui tapissent le square ont en effet été trafiquées de telle sorte qu'apparaît une fois de plus la manipulation des données et que se profile sous l'image, sous le lieu familier, 
une inquiétante étrangeté : Howard Johnson devient How Hard My Son; Panasonic Slightly Ahead of Our Time devient Paranoiac Just Lately Ahead of Our Time; Burger King est transformé en Boring Kill ; A Chorus Line et Martinique Jewellers se voient remplacés par A Border Line et Many Arty Levellers. Insérés dans les distorsions de l'image, les jeux de mots créent toutefois plus qu'un effet de double sens : ils provoquent une profonde modification du point de vue, l'expressionnisme du square nous donnant même à certains moments l'impression d'être l'objet d'hallucinations ou d'accéder à un autre niveau de conscience ». Plus loin, Ninacs (p. 22) attire l'attention sur le mot further, inscrit au centre de Sometimes Square, et qui signifie «plus », "davantage », «plus loin ». L'adverbe se pose, écrit-elle, «comme une incitation claire à chercher plus loin, comme une injonction de l'image à la fouiller davantage ». Plus loin que Paris, Montréal, Bruxelles, New York... notre parcours n'est pas fini ; nos pupilles seraient-elles en train de vivre une mutation? À force de nous y promener, avec notre regard tantôt haut perché, tantôt appliqué à déchiffrer des façades, apprendrons-nous à faire surgir de nos trajets en ville de multiples polyèdres façonnés à même un quotidien banal, mais aussi fascinants que le Diamant placé entre nos doigts au début de ce voyage?

\section{VOIR, VOYAGERIES ET VOYAGEMENTS}

Qui d'entre nous n'a pas pris plaisir à visiter des villes, à $\mathrm{y}$ inscrire ses propres chemins? Voyageries et voyagement font passer la vie et le temps, comme le dit si bien la chanson de Gilles Vigneault. Explorer sa ville ou encore une autre ville, quel que soit le continent où nous portent nos pas, comporte toujours quelque chose de fascinant. Très vite on se laisse envoûter par des couleurs, des odeurs, des formes... des foules, des personnes... des architectures fragiles ou triomphantes, délabrées ou opulentes. Ici on se sent bien; là, c'est invivable; plus loin, c'est magnifique; là-bas, il se passe des choses. Les auteurs des textes rassemblés dans ce numéro seront les guides d'un semblable voyage et les itinéraires qu'ils proposent côtoient à la fois des dangers et des ravissements.

En parcourant la rubrique Article, vous sillonnerez la planète en tous sens, dans l'espace et dans le temps. D'abord, Luc-Normand Tellier, qui a agi en qualité de conseiller sur le thème pour ce numéro, vous entraînera sous toutes les latitudes et à travers des siècles d'histoire urbaine. Vous verrez se dessiner des corridors géographiques millénaires et encore en évolution là où vous pensiez probablement ne voir que des villes éparpillées au hasard. Quand vous en saurez plus sur les maux et les espoirs des deux révolutions urbaines qu'il nous décrit, vous serez en mesure de mieux apprécier les dangers et les émerveillements qui nous attendent au début du troisième millénaire, maintenant qu'urbanisation n'est plus «synonyme de développement ». Maurice Lagueux évoquera à son tour des monuments choisis partout sur le globe pour perpétuer la mémoire des défunts et qui contribuent également à « façonner le monde » des vivants. Jean-Jacques Lavoie nous rappelle des histoires de meurtres qui nous ramènent à la nuit des temps, à la naissance des villes : celle de Romulus qui a tué son jumeau Remus et qui a fondé Rome, celle de Caïn qui a tué son frère Abel et qui a construit la première ville mentionnée dans les textes bibliques. C'est encore de meurtres dont il est question dans le texte de Françoise Naudillon. L'action se déplace à Alger.
Notre informateur est un professionnel; le danger est-il moins réel parce qu'il s'inscrit dans un univers fictif, celui des romans policiers?

Et si c'était l'âme bien plus que le corps qui était présentement en danger de mort dans nos villes? On peut se le demander en rejoignant à la rubrique Point de vue JeanPierre Bonhomme, qui a également contribué à la planification de ce numéro. Faisons route avec lui à la recherche de «l'art d'organiser la cité pour le confort psychologique». Habité par un ravissement vécu à Éphèse, il se met en quête de villes capables de "guérir l'âme ». Les villes nord-américaines que sont Montréal, Toronto, New York et Détroit réservent bien des déceptions à qui cherche ainsi la «douceur de vivre ». C'est aussi avec corps et âme que nos jeunes se cherchent une place en milieu urbain. On pourrait difficilement parler de douceur de vivre pour les jeunes de la rue, à Montréal. Les données d'Annamaria Colombo et de Michel Parazelli, présentées à la rubrique Recherche, mettent en évidence un danger imprévu : les efforts de revitalisation des centres-villes dévitalisent, disent-ils, la marge sociale juvénile et compromettent les chances de sortir de la rue.

La ville est «un lieu privilégié d'exercice du pouvoir» nous signalait déjà Pierre Delorme dans son article, faisant référence à une actualité encore bien présente à Montréal. Si la vie démocratique ne protège pas de tous les dangers, l'identité d'une ville peut néanmoins se façonner à même de constantes mutations et ne se ramène pas à des questions de dimension. En faisant un saut à la rubrique Point de vue, on peut, grâce au texte de Jean Décarie, observer presque en direct le cheminement d'une idée nouvelle dans la ville et examiner comment les réseaux d'influence, les études de faisabilité, les processus de consultation et les instances décisionnelles sont alors mobilisés. Il s'agit de proposer à des citoyens, des paroissiens, une initiative inédite à Montréal : introduire des columbariums dans les églises.

$\mathrm{Au}$ Québec, une longue tradition catholique nous avait habitués à assister souvent passivement à des rites officiels qui faisaient consensus mais, à mesure que se généralisent de nouvelles pratiques comme l'incinération, on assiste à une recherche plus intense de nouveaux modes d'expression par la ritualité, en particulier lors du décès d'un proche. Caroline Guay nous permet de constater jusqu'à quel point les choses ont changé dans les familles québécoises en citant des témoignages qu'elle a recueillis dans le cadre de sa recherche sur l'exposition des défunts à la maison. Ce récit, qui nous amène à Lévis dans la première moitié des années 1900, décrit l' " ambiance familiale » dans laquelle se déroulait alors le rite d'exposition. Des rites traditionnels aux rites plus expéditifs dictés par le rythme accéléré de la vie urbaine, c'est quand même un semblable mouvement qui se déploie, à la recherche du sens. Des voies inédites de recherche du sens s'ouvrent aussi pour apprendre à vivre avec le chagrin du deuil. Jeannette Rioux et Johanne Cadieux nous parlent, à la rubrique Regard, d'une expérience d'expression théâtrale vécue dans un espace urbain inusité : un centre culturel qui, grâce à un aménagement architectural inédit, se trouve logé en un lieu qui sert aussi de salon funéraire.

Les deux articles retenus pour la rubrique Intervention nous parlent du suicide, une tragédie qui échappe aux découpages des lieux et à la logique des tables des matières. Certes, bien des suicides ont lieu dans nos villes et ils contribuent à rendre plus criante l'urgence de redoubler d'énergie pour offrir une présence là où le danger peut devenir fascination. 
Les délires urbains peuvent probablement alimenter le mal de vivre qui pousse au suicide; la ville facilite peut-être le passage à l'acte en faisant plus vite le vide autour de la personne blessée. Le suicide nous oblige à nous demander pourquoi il nous est si difficile de nourrir la vie, en nous et autour de nous. Il nous oblige à ouvrir les yeux sur des espaces intimes désertés par cette vaste solidarité qui nous lie les uns aux autres et qui rend la vie précieuse. Le plaidoyer de Natacha Joubert pour que soit accueillie la souffrance de l'être qui choisit le suicide remet en question nos tranquilles assurances, invite les décideurs à réinvestir des énergies à la recherche d'axes d'intervention toujours plus pertinents. Dans un texte où le tableau observé est brossé sans complaisance, Jean-Dominique Leccia nous amène avec lui sur la ligne de feu, dans les urgences psychiatriques des hôpitaux parisiens. Grâce à lui, des voix que nous n'entendons pratiquement jamais nous parlent enfin : des personnes qui avaient choisi de se suicider, de ne plus faire partie de la conversation. Tant de voix, de "signaux inconnus» qui s'effacent encore plus vite quand la nuit appartient aux néons des centres-villes.

Nous ajoutons à ce numéro un article hors thème sur le cancer du sein. C'est un texte qui oblige le regard à plonger vers d'autres abîmes. La fée des Ténèbres dont nous parle Catherine Diricq semble avoir accès à tous les espaces, audelà de nos géographies urbaines. Ici, le danger se noue dans le plus intime du corps féminin et de la relation mère-fille. L'histoire clinique présentée lève le voile sur un drame mal connu : «les conséquences d'une transmission de la mère à la fille d'une pathologie à pronostic létal ». Dans ce voyage qui nous est raconté, c'est le temps qu'il faut habiter, pas à pas, bien plus que l'espace.
Comme à l'habitude, nous vous proposons aussi une bibliographie indicative et des sites Internet en complément à quelques-uns des aspects du thème abordé dans ce numéro. Vous voudrez certainement jeter un coup d'œil aux nouvelles parutions et aux comptes rendus de lectures. Nous savons que ces rubriques, même modestement placées en fin de numéro, sont avidement lues et j'en profite pour inviter ceux et celles parmi nos abonnés qui ont la plume agile à proposer des textes à l'équipe de rédaction afin de partager avec nos lecteurs leur réflexion sur des livres, des films ou des présentations théâtrales abordant le thème de la mort et du deuil.

Revenons en ville! Ces villes que nous faisons nôtres avec un bonheur jamais totalement acquis, que ce soit dans le dénuement, dans l'aisance ou dans l'opulence. Nos villes savent séduire, ravir, fasciner, nous faire la vie belle... ou banale, ou intolérable. Ce sont nos délires qui nous mettent en danger.

Nous sommes des êtres humains; nous serons toujours en danger car nous ne sommes pas éternels. Il y a sur terre une Ville éternelle, dit-on, et pourtant on y trouve des cimetières.

Ce qu'il faudrait plutôt éterniser par notre façon d'habiter nos villes, c'est un moment de ravissement.

\section{Bibliographie}

LEPAGE, Lawrence (1976). Enfin, Les Disques Bleu 1002, Canada, Éditions Vent qui vire CAPAC.

NINACS, Anne-Marie (2002). Alain Paiement: Le monde en chantier, Montréal, Galerie de l'UQÀM, 144 p.

PAIEMENT, Alain (1998). «Alain Paiement: résidence d'artiste à Bruxelles », entrevue avec Jean-Louis Godefroid, dans Contretype, ${ }^{\circ} 59$, janvier-février 1998, p. 2-9.

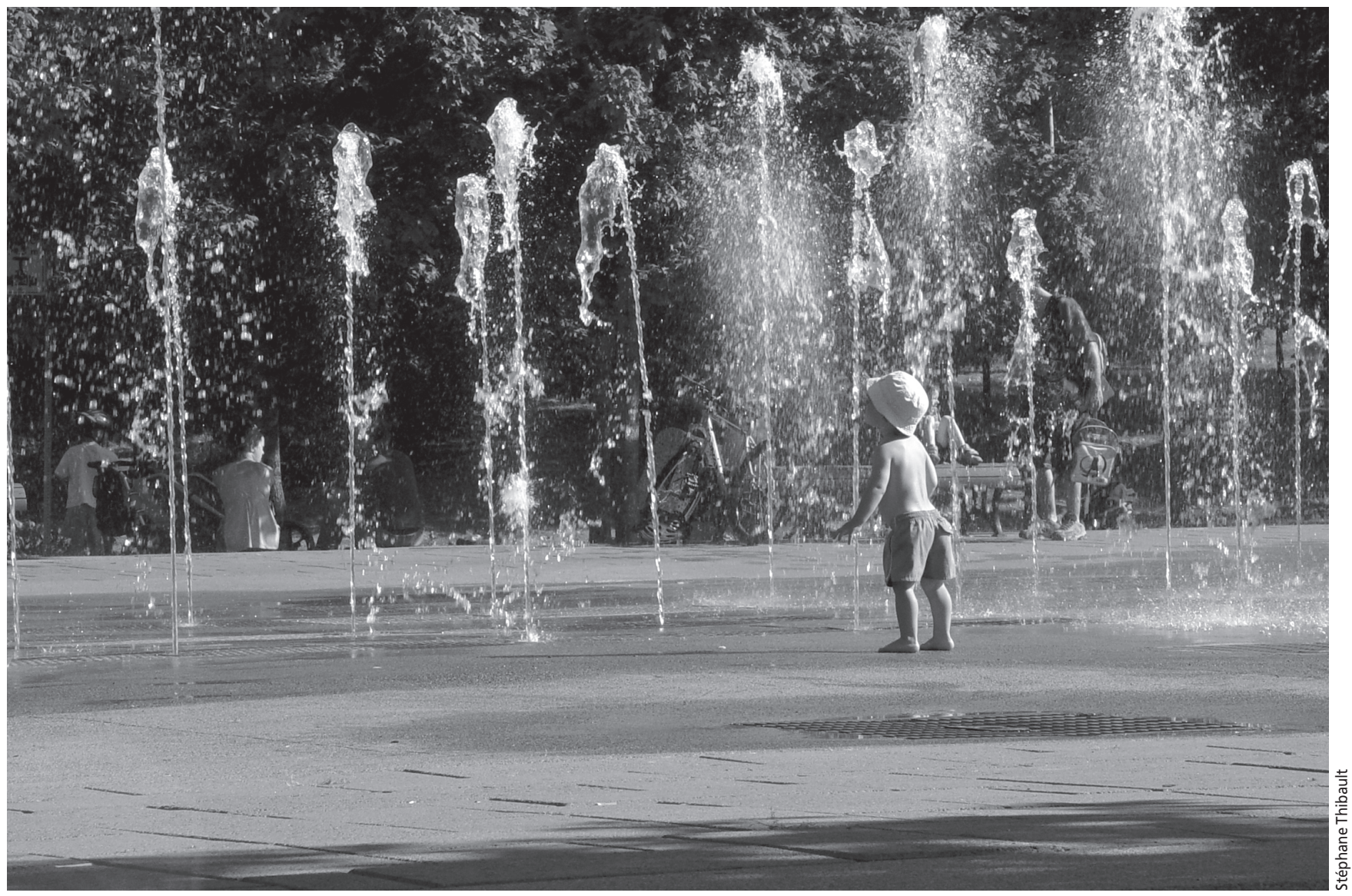

